OPEN ACCESS

Edited by:

Steve Lindemann,

Purdue University, United States

Reviewed by:

Michael A. Dubick,

United States Army Institute of

Surgical Research, United States

Ming-Hsu Chen,

University of Illinois at

Urbana-Champaign, United States

Hariom Yadav,

Wake Forest School of Medicine,

United States

*Correspondence:

Chuntian Zheng

zhengcht@163.com

Zhuang Chen

chenzhuang@agrogene.ac.cn

${ }^{\dagger}$ These authors have contributed equally to this work.

Specialty section:

This article was submitted to

Systems Microbiology,

a section of the journal

Frontiers in Microbiology

Received: 05 July 2017

Accepted: 24 October 2017

Published: 07 November 2017

Citation:

Yu T, Wang Y, Chen S, Hu M, Wang Z, Wu G, MaX, Chen Z and Zheng C

(2017) Low-Molecular-Weight

Chitosan Supplementation Increases the Population of Prevotella in the Cecal Contents of Weanling Pigs.

Front. Microbiol. 8:2182.

doi: 10.3389/fmicb.2017.02182

\section{Low-Molecular-Weight Chitosan Supplementation Increases the Population of Prevotella in the Cecal Contents of Weanling Pigs}

\author{
Ting $\mathrm{Yu}^{1,2+}$, Yu Wang ${ }^{1,3 \dagger}$, Shicheng Chen ${ }^{4 \dagger}$, Min $\mathrm{Hu}^{5}$, Zhiling Wang ${ }^{1}$, Guozhong $\mathrm{Wu}^{6}$, \\ Xianyong $\mathrm{Ma}^{1}$, Zhuang $\mathrm{Chen}^{2 *}$ and Chuntian Zheng ${ }^{1 *}$
}

${ }^{1}$ Key Laboratory of Animal Nutrition and Feed Science (South China) of Ministry of Agriculture, State Key Laboratory of Livestock and Poultry Breeding, Guangdong Public Laboratory of Animal Breeding and Nutrition, Guangdong Key Laboratory of Animal Breeding and Nutrition, Institute of Animal Science, Guangdong Academy of Agricultural Sciences, Guangzhou, China, ${ }^{2}$ Agro-Biological Gene Research Center, Guangdong Academy of Agricultural Sciences, Guangzhou, China, ${ }^{3}$ Hebei Depond Animal Health Care Science and Technology Co., Ltd, Shijiazhuang, China, ${ }^{4}$ Department of Microbiology and Molecular Genetics, Michigan State University, East Lansing, MI, United States, ${ }^{5}$ Guangdong Key Laboratory of Integrated Agro-Environmental Pollution Control and Management, Guangdong Institute of Eco-Environmental Science \& Technology, Guangzhou, China, ${ }^{6}$ Shanghai Institute of Applied Physics, Chinese Academy of Sciences, Shanghai, China

Low-molecular-weight chitosan (LC) promoted growth in weaned piglets as an alternative to feed-grade antibiotics. To investigate the influence of LC supplementation on piglets' gut microbiome and compare the differences in community composition between LC and antibiotics with $\mathrm{ZnO}$ addition, we assessed the cecal microbial community by $16 \mathrm{~S}$ rRNA gene sequencing with three treatments consisting of basal diet (CTR group), basal diet with low-molecular-weight chitosan (LC group), and basal diet with antibiotic and $\mathrm{ZnO}$ (AZ group). LC decreased $\mathrm{pH}$ more than AZ did in the cecum (both compared to CTR). Beta diversity analysis showed that community structure was distinctly different among the CTR, LC, and AZ treatments, indicating that either LC or AZ treatment modulated the piglet microbiota. Bacteroidetes, Firmicutes, and Proteobacteria dominated the community [>98\% of operational taxonomic units (OTUs)] in piglet cecal contents. Compared to CTR, both LC, and AZ increased the relative abundance of Bacteroidetes while they decreased the count of Firmicutes and AZ decreased the population of Proteobacteria. In CTR the top four abundant genera were Prevotella ( 10.4\%), Succinivibrio ( 6.2\%), Lactobacillus ( 5.6\%), and Anaerovibrio (5.4\%). Both LC and AZ increased the relative abundance of Prevotella but decreased the ratio of Lactobacillus when they compared with CTR. Moreover, LC increased the relative abundance of Succinivibrio and Anaerovibrio while $A Z$ decreased them. The microbial function prediction showed LC enriched more pathways in the metabolism of cofactors and vitamins than CTR or AZ did. LC may potentially function as an alternative to feed-grade antibiotics in weaned piglets due to its beneficial regulation of the intestinal microbiome.

Keywords: low-molecular-weight chitosan, weaned piglets, cecal microflora, microbial community, 16S rDNA sequencing 


\section{INTRODUCTION}

The piglets at an early weaning stage face a dramatic life change in the food source, the immune system as well as the environmental and social status (Pluske et al., 1997; Lallès et al., 2007). These stressful events often cause digestive disorders, nutrient malabsorption and a high incidence of diarrhea in piglets (Madec et al., 1998; Boudry et al., 2004; Fairbrother et al., 2005). Antibiotics and zinc oxide $(\mathrm{ZnO})$ have been widely supplemented into the piglet diets, which improve the growth rates and decrease the diarrhea rates (Barton, 2000; Zhu et al., 2017). However, the continuous addition of antibiotics and $\mathrm{ZnO}$ leads to negative consequences including the drug accumulation in livestock products, environmental contamination and bacterial antibiotic resistance (Barton, 2000; Vahjen et al., 2015).

Therefore, alternative diet supplements have been investigated to replace antibiotics and $\mathrm{ZnO}$ (Turner et al., 2001). Among the alternative supplements, chitosan $(\sim 1,000 \mathrm{kDa}$ of molecular weight) and its derivatives low-molecular-weight chitosan (LC or LMWC, $<150 \mathrm{kDa}$ ) or chito-oligosaccharide (COS, $<5$ $\mathrm{kDa}$ ), can be obtained from chitin after the physical, chemical, and enzymatic conversions (Yin et al., 2009). They have been widely applied in chemical, medicinal, food, and agricultural industries such as food and feed additive (Yin et al., 2009; Vinsová and Vavríková, 2011). Due to the properties of nontoxic, biocompatibility and biodegradability as the few alkaline polysaccharides with positive charge (Yin et al., 2009; Vinsová and Vavríková, 2011), they have been reported to possess many beneficial biological properties (e.g., anti-microbial, anti-tumor, anti-oxidant, anti-diabetic, anti-obesity, cholesterol lowering, immunity regulation, and metal ion adsorption in animals; Yin et al., 2009; Vinsová and Vavríková, 2011).

As the lowest molecular weight and the highest water-soluble, COS as a feed additive was more widely studied than chitosan and LC in animals (Jung et al., 2006; Han et al., 2007; Liu et al., 2008, 2010; Yang et al., 2012; Zhou et al., 2012; Kong et al., 2014; Xiao et al., 2014; Xiong et al., 2015). Several studies reported that COS $(100 \sim 1,000 \mathrm{mg} / \mathrm{kg}$ ) promoted animal growth, increased feed digestibility, reduced the incidence of diarrhea, anti-oxidative, enhanced immunity, and improved intestinal surface barrier function in weaned piglets (Jung et al., 2006; Han et al., 2007; Liu et al., 2008, 2010; Yang et al., 2012; Zhou et al., 2012; Kong et al., 2014; Xiao et al., 2014; Xiong et al., 2015). More importantly, COS protected against pathogenic infections (Escherichia coli and Staphylococcus aureus) and enhanced commensal bacteria members (lactobacilli and bifidobacteria) to maintaining the healthy gastrointestinal microflora in animals (Jung et al., 2006; Han et al., 2007; Liu et al., 2008; Yang et al., 2012; Kong et al., 2014). However, a newly reported that low dosage of COS supplementation at $30 \mathrm{mg} / \mathrm{kg}$ had no effects on promoting growth performance and even have compromised the intestinal barrier integrity (Xiong et al., 2015). The effects of LC as a feed additive on animals remain largely unknown. However, LC $(\sim 12 \mathrm{kDa})$ had much potent antimicrobial activity than did COS, including against pathogens E. coli, S. aureus, Pseudomonas aeruginosa, Salmonella enterica serovar Typhi, and Bacillus cereus (Tsai et al., 2004). Moreover, compared to that in COS treatment, diet supplementation of LC increased more lipid metabolism and intestinal disaccharidase activities in obese rats induced by high-fat-diet (Chiu et al., 2017). However, the effects of LC on microbiome profiles in piglets remain unknown. Previous information on gut microbiota affected by the probiotic LC was fragmentary and the investigations were mostly limited in the culture-based method (Tsai et al., 2004; Jung et al., 2006; Han et al., 2007; Liu et al., 2008; Yang et al., 2012; Kong et al., 2014).

Our preliminary data showed that LC $(20-30 \mathrm{kDa})$ at a dosage of $50 \mathrm{mg} / \mathrm{kg}$ improved the animal growth performance, intestinal tract health and anti-oxidant in weaned piglets (unpublished data). In this study, it was hypothesized that the diets containing LC influenced the piglet gut microbiome and might partly exhibit similar effects as in-feed antibiotics and $\mathrm{ZnO}$. High-throughput sequencing of $16 \mathrm{~S}$ rRNA gene was performed to investigate the microbial community structure variation of cecal bacteria in the weaned piglets with LC supplement and compared with that in antibiotics and $\mathrm{ZnO}$ supplement. The study helps to understand the effects of feed supplement on intestinal bacterial communities and facilitate studies of the alternative strategy for treating diarrhea in piglets. Given the similar gut bacterial communities between human beings and sows, our study here also contributes to understanding the effects of LC supplement on modulating the complexity of animal microbial communities and their functional properties in influencing health and disease.

\section{MATERIALS AND METHODS}

\section{Animals and Sample Collection}

All experimental procedures were carried out with the approval (IACUC-150701) of the Animal Care and Use Committee in Guangdong Academy of Agricultural Sciences, China. A total of 60 male weaned piglets (Duroc $\times$ Landrace $\times$ Large White) with an average weight of $6 \mathrm{~kg}$ and 21-day old were used in this study. The control (CTR) group was the piglets fed the basal diet (Supplementary Table S1); the antibiotics and $\mathrm{ZnO}$ (AZ) treatment group was fed the basal diet supplemented with aureomycin $(30 \mathrm{mg} / \mathrm{kg})$, polymyxin $\mathrm{E}(12 \mathrm{mg} / \mathrm{kg})$ and $\mathrm{ZnO}$ $(3,000 \mathrm{mg} / \mathrm{kg})$; the LC group was those given the basal diet added with low-molecular-weight (20,000 30,000 Da) chitosan (50 mg/kg), which was provided by Jiaxing Korui Biotech Co. Ltd, Zhejiang, China (http://www.korui-china.com/). The product of LC (KR901, Korui) is fabricated by physical methods and in the form of fine powder, water insoluble but soluble in dilute acidic solution, and recommended dosage $50 \mathrm{~g} / \mathrm{T}$ for piglet's feed. Five replicates were used for each treatment (4 piglets per replicate, totaling 20 animals). The feeding trials lasted for 28 days. The food and water were daily processed ad lib. At day 28, 1 randomly selected piglet in individual replicate for each treatment (total 5 animals/treatment) were slaughtered under anesthesia. The contents of cecum were collected and proceeded. The cecal $\mathrm{pH}$ was immediately determined.

\section{DNA Extraction, Library Construction and 16S rDNA Sequencing}

Microbial genomic DNA was extracted from $200 \mathrm{mg}$ of the sample using the QIAamp DNA stool minikit (Qiagen, 
Germany) according to the manufacturer's recommendation. DNA quality was evaluated by the agarose gel electrophoresis. The V4 hypervariable regions (the forward primer was $520 \mathrm{~F}$ 5-AYTGGGYDTAAAGNG-3 and the reverse primer was $802 \mathrm{R}$ 5-TACNVGGGTATCTAATCC-3) of $16 \mathrm{~S}$ rDNA were PCR amplified from microbial genomic DNA (Caporaso et al., 2011). Briefly, $2 \mu \mathrm{L}$ of diluted DNA sample $(\sim 20 \mathrm{ng} / \mu \mathrm{L})$ was used for PCR amplification ( $25 \mu \mathrm{L}$ mixtures). The PCR conditions were as follows: one pre-denaturation cycle at $98^{\circ} \mathrm{C}$ for $2 \mathrm{~min}, 25$ cycles of denaturation at $98^{\circ} \mathrm{C}$ for $15 \mathrm{~s}$, annealing at $50^{\circ} \mathrm{C}$ for $30 \mathrm{~s}$, and elongation at $72^{\circ} \mathrm{C}$ for $30 \mathrm{~s}$, and one post-elongation cycle at $72^{\circ} \mathrm{C}$ for $5 \mathrm{~min}$. The PCR amplicon products were purified using $2 \%$ agarose gels and used to construct the sequencing library. The libraries of amplicons were attached to Illumina sequencing adapters using the NEBNext Ultra ${ }^{\mathrm{TM}}$ II DNA Library Prep Kit for Illumina (E7645L), purified using AMPure XP beads (Biomek, USA) and quality controlled on an Agilent 2100 Bioanalyzer (Agilent, USA). The pooled libraries were pair-end sequenced on the Illumina MiSeq platform with using $2 \times 250$ bp MiSeq reagent kit v2 (Illumina, USA).

\section{DNA Sequence Analysis}

Raw sequences were filtered with the average base quality lower than $\mathrm{Q}_{20}$, the quality of the head or tail bases lower than $\mathrm{Q}_{20}$, sequence lengths shorter than $150 \mathrm{bp}$, or reads with $\mathrm{N}$. Then FLASH (v1.2.7) was used to assemble the paired-end sequences (Magoc and Salzberg, 2011). Reads with homopolymer runs exceeding $8 \mathrm{bp}$, primer mismatches, ambiguous bases and sequence lengths shorter than $150 \mathrm{bp}$ were further removed in QIIME (v1.9.0) (Caporaso et al., 2010). The UCHIME (Chiu et al., 2017) of mothur (v1.31.2) (Edgar et al., 2011) was used to remove the chimera sequences.

Operational taxonomic units (OTUs) were counted for all samples with a cutoff of $97 \%$ identity using the UCLUST function (Schloss et al., 2009) in QIIME. Any reads < 7 times $(0.001 \%)$ were removed to minimize the impact of rare OTUs on our data analysis (Edgar and Baterman, 2010). The representative sequences of each clustered OTU were selected according to the maximum length, aligned to Greengenes 16S rRNA gene database (v13.8) (Bokulich et al., 2013) and classified by RDP classifier (v.2.2) (Mc Donald et al., 2012). The alpha-diversity indices (PD, Shannon, observed species and Chaol) were calculated for each sample, and beta-diversity (non-metric multidimensional scaling, NMDS) analysis was performed to show the group differences in microbial community structure. Betadiversity statistical analyses were also tested using permutational multivariate analysis of variance (PERMANOVA) based on BrayCurtis dissimilarities and 999 permutations in the vegan package (v. 2.3.2) (Wang et al., 2007).

\section{Microbial Function Prediction and Statistical Analysis}

The microbial function was predicted using Phylogenetic Investigation of Communities by Reconstruction of Unobserved States (PICRUSt) (v1.1.0) (Oksanen et al., 2015). Based on the pre-calculated Greengenes (v13.5) database (Langille et al., 2013), PICRUSt was performed on the abundance predictions of
Kyoto Encyclopedia of Genes and Genomes (KEGG) orthologs and KEGG pathways of bacterial communities. The functional differences among groups were compared using Statistical Analysis of Metagenomic Profiles (STAMP) (Parks et al., 2014). Statistical analysis of two groups was conducted for Two-sided Welch's $t$-test and Benjamini-Hochberg FDR correction. Multiple groups were conducted for ANOVA with Tukey-Kramer test and Benjamini-Hochberg correction. Differences were considered significant at $P<0.05$. Heatmap diagrams were plotted in $\mathrm{R}$ environment (v3.1.2) (http://www.r-project.org).

\section{Sequence Data Accession Number}

Raw paired-end reads were submitted to the Sequence Read Archive of the NCBI (accession number: SRP104359).

\section{RESULTS}

\section{Effects of LC or AZ on the pH in Cecal Samples}

The cecal $\mathrm{pH}$ value in the piglets fed with LC was determined to be 6.19 , which was significantly lower than that in the control (6.55) $(P<0.05)$. Similarly, compared to that in the control, the cecal $\mathrm{pH}$ (6.33) was significantly lower in the piglets fed with $\mathrm{AZ}(P<0.05)$. The $\mathrm{pH}$ between $\mathrm{LC}$ and $\mathrm{AZ}$ treatments was not significantly different $(P>0.05)$ (Supplementary Table S2).

\section{Characteristics of Midgut Bacteria Community Libraries}

At least 47,083 sequence reads were obtained per sample and a total of 706,251 high-quality sequences were used for later analysis (Supplementary Table S3). Based on 97\% sequence similarity, these sequences were assigned to 17,890 OTUs. After removing the rare OTUs (lower than $0.001 \%$ of total sequences), 2,242, 2,134, and 2,129 OTUs were retained from CTR group, LC group, and AZ group, respectively (Supplementary Table S4). From the Veen analysis of OTU, out of total 2,624 OTUs, 1,585 ( $\sim 60 \%$ of the total OTUs) existed in three groups (Figure 1A). Instead, 148, 72, and 108 unique OTUs were identified in CTR, LC, and AZ groups, respectively.

\section{Effects of Dietary LC and AZ on Alpha and Beta Bacterial Diversity}

We compared bacterial diversity (PD and Shannon index) and richness (observed species and Chao index) indices for alphadiversity. $\mathrm{PD}$ index in the LC group or AZ group was significantly lower than that in the CTR group $(P<0.05)$, showing that the bacterial diversity in cecum was decreased by AZ or LC (Figure 1B). Shannon index between the two treatments did not show statistical difference while the value in the treatments was lower than the control (Supplementary Table S5). However, there was no significant difference in diversity index (PD and Shannon index) between LC and AZ $(P>0.05)$, indicating both of the treatment strategies decreased bacterial diversity under our testing conditions. About community richness indices (Observed species and Chao), there was no significant difference between LC and CTR, indicating that LC did not affect bacterial richness. However, the number of observed species or Chao index in the 


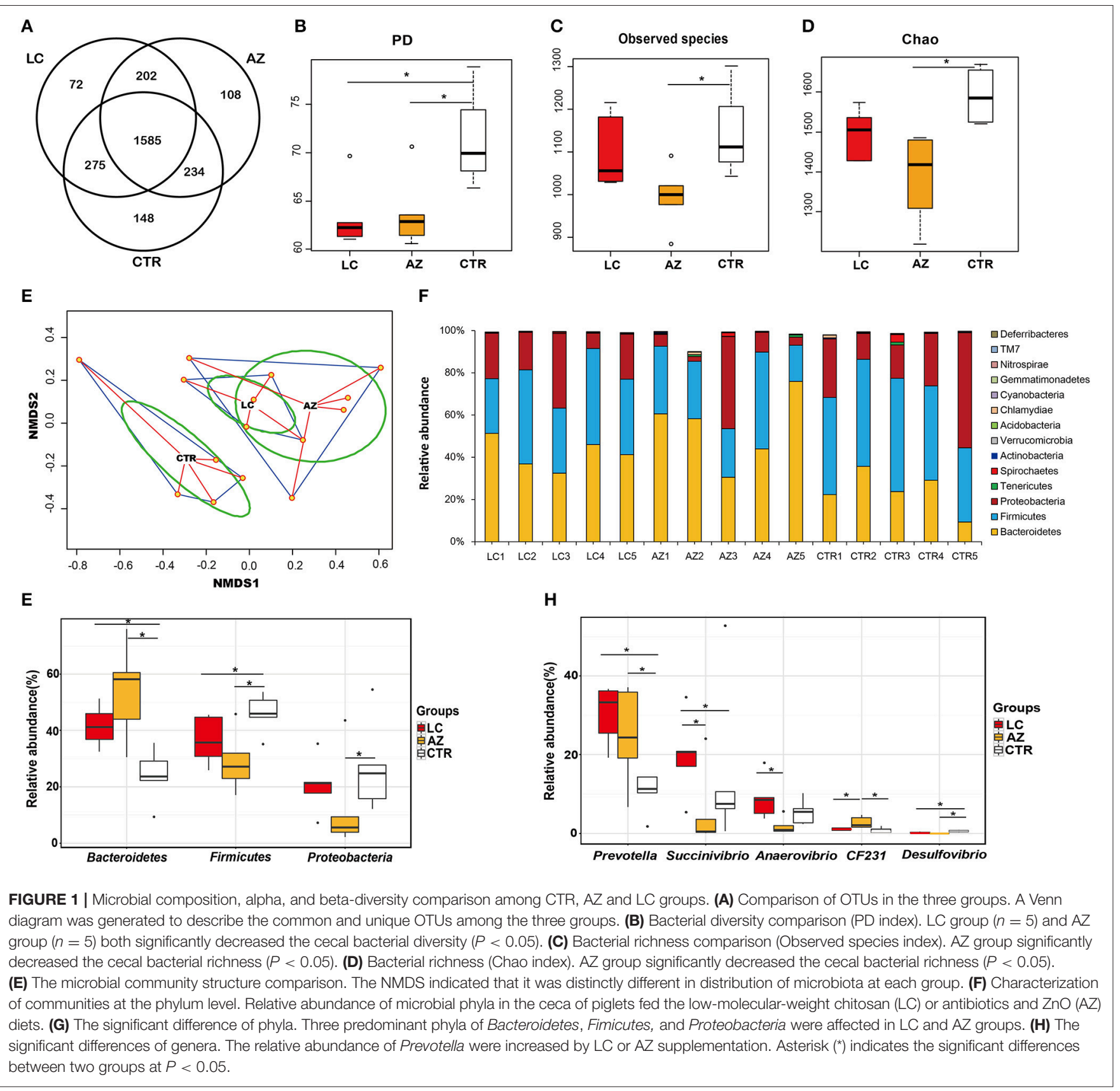

AZ group was significantly lower than that in CTR $(P<0.05)$ (Figures 1C,D), indicating that AZ decreased bacterial richness in the cecum. Collectively, our data suggested that piglets fed AZ had a decreased bacterial richness and diversity. Additionally, supplementary LC only decreased the gut bacterial diversity rather than bacterial richness.

For beta diversity analysis, we examined the relationships in cecal microbiome between the control, AZ supplement and LC addition using NMDS. The distribution of microbiota at each group was distinctly clustered separately along principal coordinate (Figure 1E). Moreover, PERMANOVA was used to test the Beta-diversity statistical analysis. The results showed that three groups were distinctly different in the distribution of microbiota $(P<0.05)$ (Table 1$)$, indicating that LC and AZ significantly affected the cecal bacterial structure when compared to CTR.

\section{Effects of Dietary LC and AZ on the Composition of the Cecal Microbiota}

Twenty-two bacterial phyla were assigned in the LC, AZ, and CTR groups and 14 of them were shared among them (Supplementary Table S6). Bacteroidetes, Firmicutes, and Proteobacteria accounted for more than $98 \%$ of the total sequence reads (Figure 1F). Approximately 1\% of sequences in the cecum samples could not be assigned to a certain 
TABLE 1 | Pseudo F table of PERMANOVA analysis based on Bray-Curtis dissimilarities.

\begin{tabular}{|c|c|c|c|c|}
\hline Source of variance & Degree of freedom & $\boldsymbol{F}$ & $R^{2}$ & Padjusted \\
\hline Groups $^{a}$ & 2 & 2.813 & 0.319 & $0.002^{\star}$ \\
\hline Pairwise comparison & $\boldsymbol{F}$ & $R^{2}$ & $\boldsymbol{P}$ & Padjusted \\
\hline LC vs. CTR ${ }^{a}$ & 3.157 & 0.283 & 0.009 & $0.027^{\star}$ \\
\hline AZ vs. CTR ${ }^{a}$ & 2.689 & 0.252 & 0.009 & $0.027^{\star}$ \\
\hline LC vs. $A Z^{a}$ & 2.597 & 0.245 & 0.031 & 0.093 \\
\hline
\end{tabular}

a Based on genus level and $n=5$ per group.

*Significant $P$-values $(<0.05)$ are bolded.

rank (unclassified) (Supplementary Table S6), suggesting that there were uncharacterized bacterial taxa existing in the cecal microbiome. The proportion of Bacteroidetes in LC and AZ was 1.5- and 1.9-fold higher than that in the CTR group $(P<0.05)$, respectively (Figure 1G). Instead, the proportion of Firmicutes in the LC and AZ treatments was around 0.8- and 0.6-fold lower than that in the CTR group $(P<0.05)$, respectively (Figure 1G). The proportion of Proteobacteria in the AZ treatment was 0.2fold lower than that in the CTR group $(P<0.05)$, but in the LC treatment, it was in accordance with CTR group (Figure 1G).

Moreover, 41 bacterial classes (Supplementary Table S7), 68 orders (Supplementary Table S8), 96 families (Supplementary Table S9), and 124 genera (Supplementary Table S10) were assigned to all the three treatments. On the genus level, the relative abundance of Prevotella in the LC group (30\% of the total bacterial community) and AZ group (29\%) was significantly higher than that in the CTR group $(10 \%)(P<0.05)$ (Figure $\mathbf{1 H})$. Compared to CTR and AZ groups, the relative abundance of Succinivibrio was significantly increased by LC treatment $(P<0.05)$ (Figure 1H). Similarly, the relative abundance of Anaerovibrio in the LC group was significantly higher than that in $\mathrm{AZ}$ group $(P<0.05)$ (Figure $\mathbf{1 H})$. However, the relative abundance of CF231 in AZ group was significantly higher than that in CTR as well as LC $(P<0.05)$ (Figure 1H). The relative abundance of Desulfovibrio in treatments LC and AZ was significantly lower than that in the control group $(P<0.05)$ (Figure 1H).

\section{Function Prediction of the Microbial Community in the Cecum}

Given the distinct microbiome changes within the LC and AZ pigs compared to CTR pigs, we tested whether the different treatments would lead to functional changes in each microbiome. Prediction of metagenome functional contents was conducted by applying PICRUSt to insight our $16 \mathrm{~S}$ rDNA sequences. Compared to CTR, both LC and AZ had significantly higher function enrichments of energy metabolism, metabolism of terpenoids and polyketides, digestive systems and cell growth and death $(P<0.05)$ (Figures 2A,B). However, both treatments dramatically reduced the function enrichment of environmental information processing such as membrane transport $(P<0.05)$ (Figures 2A,B). In addition, LC group significantly increased the abundance of glycan biosynthesis and metabolism when compared to that in CTR group $(P<0.05)$ (Figure 2A). The relative abundance in the metabolism of cofactors and vitamin in LC treatment was higher than that in CTR or AZ group $(P<0.05)$ (Figures 2A,C). Furthermore, we also analyzed the microbial genes connected to KEGG Orthology (KO) terms. 1,373 of $6,014 \mathrm{KO}$ terms (23\%) were found to be significantly different among AZ, LC, and CTR groups based on TukeyKramer's ANOVA Test $(P<0.05)$. Remarkably, out of 1,373 , $462 \mathrm{KO}$ terms were assigned to bacteria Prevotella. It is worthy of highlighting that most of them (462 different KO terms from any two-group comparisons) were enriched on carbohydrate metabolism (15\% different $\mathrm{KO}$ terms), metabolism of cofactors and vitamins (9\%), energy metabolism (8\%), glycan biosynthesis and metabolism (6\%) (Figure 3), suggesting that the drastically increased Prevotella by LC and AZ played an important function among the intestinal microbiota. Further, 79 different KO terms whose relative abundance were $>0.1 \%$ in any group were screened out, and most of them including iron complex were increased in LC than in CTR group (Figure 4). In the metabolism pathways, there were 62 and 29 different KO terms, whose relative abundance was $>0.01 \%$ in any group, involved in the metabolism of cofactors and vitamins (Supplementary Figure S1) and glycan biosynthesis and metabolism (Supplementary Figure S2), respectively.

\section{DISCUSSION}

The gut microbiota plays a critical role in animal growth and production; a different influence on microbiome profiles resulting from different treatments is expected to impact host digestion efficiency and intestinal function (Frese et al., 2015). LC, a growth promoter as the alternative supplement in animals, possibly promoted the animal growth performance by modulating the microbiota in the host digestion tract. Our study showed that supplementation of LC $(50 \mathrm{mg} / \mathrm{kg})$ to piglets decreased the cecal bacterial diversity without affecting bacterial richness while utilization of antibiotics and $\mathrm{ZnO}$ as antimicrobials decreased the cecal bacterial community richness and the bacterial diversity (Supplementary Table S5 and Figures 1B-D). Our early study also observed that in-feed antibiotics or high dietary $\mathrm{ZnO}$ supplementation to weaned piglets increased the microbiota diversity and richness of ileal digesta whereas decreased the microbiota diversity of the colonic digesta (Yu et al., 2017). Further, microbial community structure in piglets fed with LC was remarkably different from that in AZ group or CTR (Table 1, Figure 1E). Collectively, our results demonstrated LC differently modulated the intestinal microbiota in piglets using a different mechanism from that in $\mathrm{AZ}$.

In this study, the supplementation of LC $(50 \mathrm{mg} / \mathrm{kg})$ to weaned piglets significantly increased the cecal relative abundance of Bacteroidetes (Figure 1G). Bacteroidetes are wellknown excellent degraders for plant polysaccharides and other recalcitrant organic carbon and nitrogen sources (Salyers, 1984). It is also reported that abundant Bacteroidetes may prevent diarrhea because members of phylum Bacteroidetes are known to interact with the gut immune system, suggesting a link between early microbiota colonization and gut immune maturation after weaning (Jakobsson et al., 2013). Particularly, the abundance 


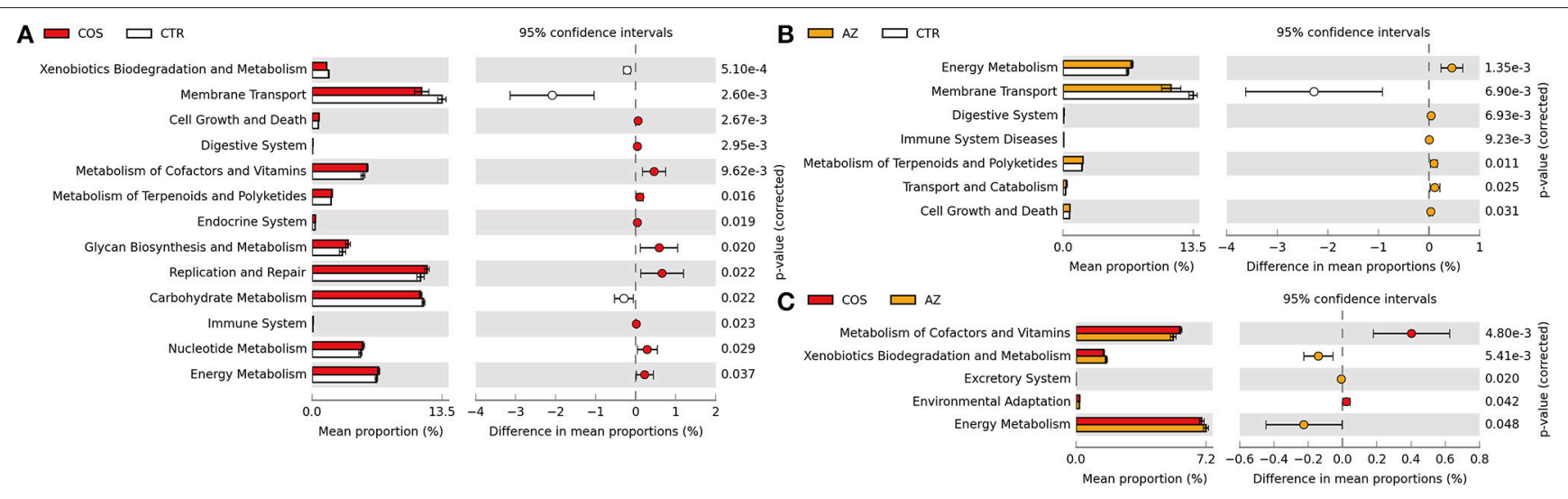

FIGURE 2 | Comparison of microbial function prediction. PICRUSt-predicted relative abundance of KEGG pathway (KEGG level 2) was compared among LC, AZ, and CTR groups. (A) the difference of the predicted function between LC and CTR; (B) the difference of the predicted function between AZ and CTR; (C) the difference of the predicted function between LC and AZ. Statistical analysis was conducted using a Welch's $t$-test between two groups; a significant difference of KEGG pathways (P.adj < 0.05) was displayed.

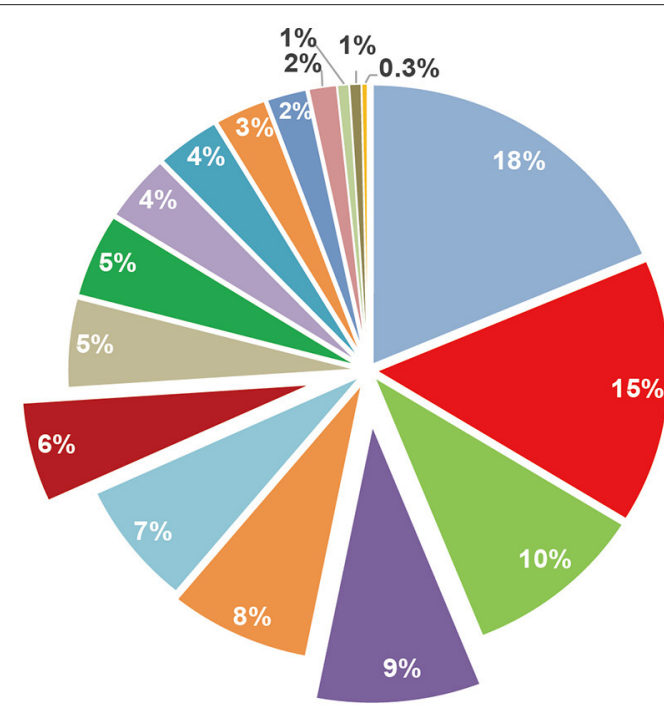

\author{
- Translation (18\%) \\ - Carbohydrate metabolism (15\%) \\ - Nucleotide metabolism (10\%) \\ - Metabolism of cofactors and vitamins (9\%) \\ - Energy metabolism (8\%) \\ - Amino acid metabolism (7\%) \\ - Glycan biosynthesis and metabolism (6\%) \\ - Replication and repair (5\%) \\ - Folding, sorting and degradation (5\%) \\ - Metabolism of terpenoids and polyketides (4\%) \\ - Membrane transport (4\%) \\ - Lipid metabolism (3\%) \\ - Metabolism of other amino acids (2\%) \\ - Cell growth and death (2\%) \\ - Drug resistance (1\%) \\ - Endocrine system (1\%) \\ - Digestive system (0.3\%)
}

FIGURE 3 | Different KO terms in Prevotella. The abundance of enrichments was shown: Translation (18\%), Carbohydrate metabolism (15\%), Nucleotide metabolism (10\%), Metabolism of cofactors and vitamins (9\%), Energy metabolism (8\%), Amino acid metabolism (7\%), Glycan biosynthesis and metabolism (6\%).

of genus Prevotella within phylum Bacteroidetes was acutely increased from $10.41 \%$ in control group to $30.17 \%$ in LC group (Supplementary Table S10 and Figure 1H). Prevotella are saccharolytic and produce acetate and succinic acids as end fermentation products (Downes et al., 2007). Evidence showed a strong association between Prevotella and carbohydrates from fiber-rich diets or from long-term carbohydrates diets (De Filippo et al., 2010; Wu et al., 2011). It was also reported that a higher abundance of Prevotellaceae dominated in fecal microbiota of healthy piglets when compared to post-weaning diarrheic piglets (Dou et al., 2017). Thus, diets supplementation of LC, as oligosaccharides that belong to carbohydrates, stimulated the amount increase of Bacteroidetes and Prevotella to help the breakdown of carbohydrate and maybe improve the intestinal immune and decrease diarrhea. Further investigations are needed to establish the relation between the abundance of Bacteroidetes or Prevotella and diarrhea. Kong et al. (2014) indicated that dietary supplementation of COS at $500 \mathrm{mg} / \mathrm{kg}$ increased the number of Prevotella in ileal contents (Kong et al., 2014). Our study here showed that LC supplementation (50 $\mathrm{mg} / \mathrm{kg}$ ) could increase the gastrointestinal Prevotella population. Moreover, antibiotics and $\mathrm{ZnO}$ supplement also increased the relative abundance of Prevotella. The weaned pigs fed with chlortetracycline had an increased Prevotella in fecal samples (Li et al., 2017). Collectively, Prevotella could be a potential microbial marker for modulation effects of gut microflora by $\mathrm{LC}$ or $\mathrm{AZ}$ supplementation.

An interesting finding is that the relative abundance of Succinivibrio was increased 38-fold in LC group while it was decreased 0.17-fold in AZ group compared to that in CTR 


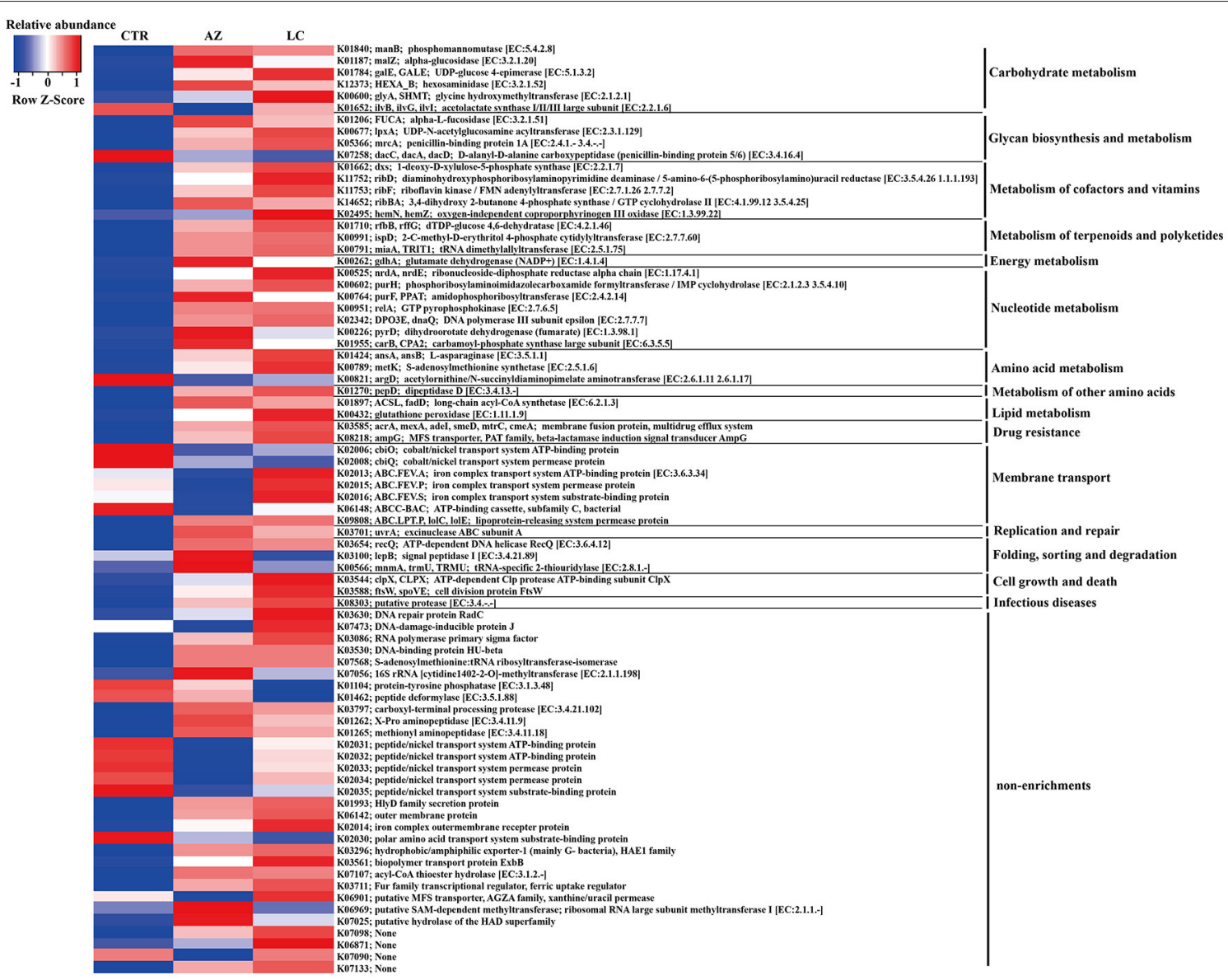

FIGURE 4 | Seventy-nine different $\mathrm{KO}$ terms. The relative abundance greater than $0.1 \%$ in any group was shown.

(Figure 1H). The relative abundance of Succinivibrio in faece was decreased in the weaned pigs fed with chlortetracycline, or zinc bacitracin, or colistin sulfate, or a mixture of them, indicating that Succinivibrio was sensitive to antibiotics or zinc (Li et al., 2017). Bacteria Succinivibrio are known to degrade starch and produce acetic and succinic acids (Wang et al., 2017). Our results further demonstrated that Succinivibrio involving in polysaccharide biodegradation can be increased by some oligosaccharides (i.e., COS). Moreover, the relative abundance of Enterobacteriaceae and Staphylococcus in the cecum presented a decreased trend in LC group and AZ group (Supplementary Tables S9, S10). However, dietary supplementation with LC $(50 \mathrm{mg} / \mathrm{kg})$ and AZ group had no effects on increasing Lactobacillus and Bifidobacterium counts in the cecum (Table S10). The decreased intestinal $\mathrm{pH}$ may contribute to inhibit intestinal pathogen propagation and alleviate post-weaning diarrhea in young animals (Mourao et al., 2006). The cecal pH in LC group was the lowest compared to AZ and CTR (Supplementary Table $\mathrm{S} 2$ ). It is reasonable to assume the decreased $\mathrm{pH}$ is attributed to the increased Prevotella and Succinivibrio in LC because both produce acetic and succinic acids as the end fermentation products (Downes et al., 2007; Wang et al., 2017).
Prediction of metagenome functional contents showed that several metabolic pathways were enriched in piglets with LC supplementation including energy metabolism, metabolism of terpenoids and polyketides, digestive systems, cell growth and death, glycan biosynthesis and metabolism as well as metabolism of cofactors and vitamin (Figure 2). It is worthy of highlighting that "digestive systems," particularly those involved in biodegradation of plant materials (Figure 4), are very important for early-weaned piglets because of the sudden switching from protein-rich milk to plant fiber-rich diets. Abbreviation of such pathways in animal digestion tracts will cause digestive disorders, nutrient malabsorption and a high incidence of diarrhea. Terpenoids and polyketides serve numerous biochemical functions such as quinones in electron transport chains, membrane components, hormones, anti-microbial, and anti-parasites.

Moreover, LC increased the abundances of riboflavin $\left(\mathrm{V}_{\mathrm{B} 2}\right)$ kinase and iron complex related proteins (Figure 4). LC effectively chelates some vitamins and heavy metals (such as $\mathrm{Fe}^{3+}$ ) (Varma et al., 2004; Sun et al., 2010), which may contribute to solubilize these cofactors and transport them to hosts. 
In conclusion, low-molecular-weight chitosan modulated the gut microbial diversity and altered the microbial community in the cecum of weaned piglets, and showed comparable effects to in-feed antibiotics and $\mathrm{ZnO}$ additive, especially increased the population of Prevotella microbiota in the cecal digesta. The understanding on effects of low-molecular-weight chitosan on intestinal bacterial communities may provide insights into future application of the alternative strategy for treating diarrhea in piglets.

\section{AUTHOR CONTRIBUTIONS}

$\mathrm{CZ}$ and $\mathrm{ZC}$ conceived and designed the experiments; YW performed the experiments; TY and SC analyzed the data; $\mathrm{MH}$, ZW and XM provided the experimental materials; GW funded the sequencing cost. TY and SC wrote the manuscript. All authors reviewed the manuscript.

\section{REFERENCES}

Barton, M. D. (2000). Antibiotic use in animal feed and its impact on human health. Nutr. Res. Rev. 13, 279-299. doi: 10.1079/095442200108729106

Bokulich, N. A., Subramanian, S., Faith, J. J., Gevers, D., Gordon, J. I., Knight, R., et al. (2013). Quality-filtering vastly improves diversity estimates from Illumina amplicon sequencing. Nat. Methods 10, 57-59. doi: 10.1038/nmeth. 2276

Boudry, G., Péron, V., Huërou-Luron, I., Lallès, J. P., and Sève, B. (2004). Weaning induces both transient and long-lasting modifications of absorptive, secretory, and barrier properties of piglet intestine. J. Nutr. 134, 2256-2262.

Caporaso, J. G., Kuczynski, J., Stombaugh, J., Bittinger, K., Bushman, F. D.,Costello, E. K., et al. (2010). QIIME allows analysis of highthroughput community sequencing data. Nat. Methods 7, 335-336. doi: 10.1038/nmeth.f.303

Caporaso, J. G., Lauber, C. L., Walters, W. A., Berg-Lyons, D., Lozupone, C. A., Turnbaugh, P. J., et al. (2011). Global patterns of 16S rRNA diversity at a depth of millions of sequences per sample. Proc. Natl. Acad. Sci. U.S.A. 108, 4516-4522. doi: 10.1073/pnas.1000080107

Chiu, C. Y., Feng, S. A., Liu, S. H., and Chiang, M. T. (2017). Functional comparison for lipid metabolism and intestinal and fecal micro?ora enzyme activities between low molecular weight chitosan and chitosan Oligosaccharide in high-fat-diet-fed rats. Mar. Drugs 15:E234. doi: 10.3390/md150 70234

De Filippo, C., Cavalieri, D., Di Paola, M., Ramazzotti, M., Poullet, J. B., Massart, S., et al. (2010). Impact of diet in shaping gut microbiota revealed by a comparative study in children from Europe and rural Africa. Proc. Natl. Acad. Sci. U.S.A. 107, 14691-14696. doi: 10.1073/pnas.1005963107

Dou, S., Gadonna-Widehem, P., Rome, V., Hamoudi, D., Rhazi, L., Lakhal, L., et al. (2017). Characterisation of early-life fecal microbiota in susceptible and healthy pigs to post-weaning diarrhoea. PLOS ONE 12:e0169851. doi: 10.1371/journal.pone.0169851

Downes, J., Sutcliffe, I. C., Booth, V., and Wade, W. G., (2007). Prevotella maculosa sp. nov., isolated from the human oral cavity. Int. J. Syst. Evol. Microbiol. 57, 2936-2939. doi: 10.1099/ijs.0.65281-0

Edgar, R. C., and Baterman, A. (2010). Search and clustering orders of magnitude faster than BLAST. Bioinformatics 26, 2460-2461. doi: 10.1093/bioinformatics/btq461

Edgar, R. C., Haas, B. J., Clemente, J. C., Quince, C., and Knight, R. (2011). UCHIME improves sensitivity and speed of chimera detection. Bioinformatics 27, 2194-2200. doi: 10.1093/bioinformatics/btr381

Fairbrother, J. M., Nadeau, E., and Gyles, C. L. (2005). Escherichia coli in postweaning diarrhea in pigs: an update on bacterial types, pathogenesis, and prevention strategies. Anim. Health Res. Rev. 6, 17-39. doi: 10.1079/AHR2005105

\section{ACKNOWLEDGMENTS}

This project was supported by the National Science-technology Support Plan (2012BAD39B01-5) and National modern agriculture industry technology system (CARS-36), Natural Science Foundation of Guangdong Province (2015A030310368), Presidential Foundation of the Guangdong Academy of Agricultural Sciences (201427). Science and Technology Project of Guangdong Province (2009B090200067, 2013A061401020, 2016A020210041, 2016B070701013).

\section{SUPPLEMENTARY MATERIAL}

The Supplementary Material for this article can be found online at: https://www.frontiersin.org/articles/10.3389/fmicb. 2017.02182/full\#supplementary-material

Frese, S. A., Parker, K., Calvert, C. C., and Mills, D. A. (2015). Diet shapes the gut microbiome of pigs during nursing and weaning. Microbiome 3:28. doi: 10.1186/s40168-015-0091-8

Han, K. N., Kwon, I. K., Lohakare, J. D., Heo, S., and Chae, B. J. (2007). Chitoolligosaccharides as an alternative to antimicrobials in improving performance, digestibility and microbial ecology of the gut in weanling pigs. Asian-Aust. J. Anim. Sci. 20, 556-562. doi: 10.5713/ajas.2007.556

Jakobsson, H. E., Abrahamsson, T. R., Jenmalm, M. C., Harris, K., Quince, C., Jernberg, C., et al. (2013). Decreased gut microbiota diversity, delayed Bacteroidetes colonisation and reduced Th1 responses in infants delivered by Caesarean section. Gut 63:4. doi: 10.1136/gutjnl-2012-493 303249

Jung, B. O., Kim, B. R., Park, H. J., Oh, D. Y., and Chung, S. J. (2006). Antimicrobial activities of chitooligosaccharide and water-soluble chitosan. $J$. Chitin. Chitosan. 11, 108-112.

Kong, X. F., Zhou, X. L., Lian, G. Q., Blachier, F., Liu, G., Tan, B. E., et al. (2014). Dietary supplementation with chitooligosaccharides alters gut microbiota and modifies intestinal luminal metabolites in weaned Huanjiang mini-piglets. Livest. Sci. 160, 97-101. doi: 10.1016/j.livsci.2013.11.023

Lallès, J. P., Bosi, P., Smidt, H., and Strokes, C. R. (2007). Weaning a challenge to gut physiologists. Livest. Sci. 108, 82-93. doi: 10.1016/j.livsci.2007.01.091

Langille, M. G. I., Zaneveld, J., Caporaso, J. G., McDonald, D., Knights, D., Reyes, J. A., et al. (2013). Predictive functional profiling of microbial communities using 16S rRNAmarker gene sequences. Nat. Biotechnol. 31, 814-821. doi: 10.1038/nbt.2676

Li, H., Liang, T., Chu, Q., Xu, F., Li, Y., Fu, L., et al. (2017). Effects of several in-feed antibiotic combinations on the abundance and diversity of fecal microbes in weaned pigs. Can. J. Microbiol. 63, 402-410. doi: 10.1139/cjm-201 6-0681

Liu, P., Piao, X. S., Kim, S. W., Wang, L., Shen, Y. B., Lee, H. S., et al. (2008). Effects of chito-oligosaccharide supplementation on the growth performance, nutrient digestibility, intestinal morphology, and fecal shedding of Escherichia coli and Lactobacillus in weaning pigs. J. Anim. Sci. 86, 2609-2618. doi: 10.2527/jas.2007-0668

Liu, P., Piao, X. S., Thacker, P. A., Zeng, Z. K., Li, P. F., Wang, D., et al. (2010). Chito-oligosaccharide reduces diarrhea incidence and attenuates the immune response of weaned pigs challenged with Escherichia coli K88. J. Anim. Sci. 88, 3871-3879. doi: 10.2527/jas.2009-2771

Madec, F., Bridoux, N., Bounaix, S., and Jestin, A. (1998). Measurement of digestive disorders in the piglet at weaning and related risk factors. Prev. Vet. Med. 35, 53-72. doi: 10.1016/S0167-5877(97)00057-3

Magoc, T., and Salzberg, S. L. (2011). FLASH: fast length adjustment of short reads to improve genome assemblies. Bioinformatics 27, 2957-2963. doi: 10.1093/bioinformatics/btr507

Mc Donald, D., Price, M. N., Goodrich, J., Nawrocki, E. P., De Santis, T. Z., Probs, A., et al. (2012). An improved greengenes taxonomy with explicit ranks 
for ecological and evolutionary analyses of bacteria and archaea. ISME J. 6, 610-618. doi: 10.1038/ismej.2011.139

Mourao, J. L., Pinheiro, V., Alves, A., Guedes, C. M., Pinto, L., Saavedra, M. J., et al. (2006). Effect of mannanoligosaccharides on the performance, intestinal morphology and cecal fermentation of fattening rabbits. Anim. Feed Sci. Technol 126, 107-120. doi: 10.1016/j.anifeedsci.2005.06.009

Oksanen, J., Blanchet, F. G., Kindt, R., Legendre, P., Minchin, P. R., and O'Hara, R. B. (2015). Vegan: Community Ecology. Available online at: http://cran.r-project. org/package $=$ vegan

Parks, D. H., Tyson, G. W., Hugenholtz, P., and Beiko, R. G. (2014). STAMP: statistical analysis of taxonomic and functional proflies. Bioinformatics 30, 3123-3124. doi: 10.1093/bioinformatics/btu494

Pluske, J. R., Hampson, D. J., and Williams, I. H. (1997). Factors influencing the structure and function of the small intestine in the weaned pig: a review. Livest. Prod. Sci. 51, 215-236. doi: 10.1016/S0301-6226(97)0 0057-2

Salyers, A. A. (1984). Bacteroides of the human lower intestinal tract. Annu. Rev. Microbiol. 38, 293-313.

Schloss, P. D.,Westcott, S. L., Ryabin, T., Hall, J. R., Hartmann, M., Hollister, E. B., et al. (2009). Introducing mothur: open-source, platformindependent, community-supported software for describing and comparing microbial communities. Appl. Environ. Microbiol. 75, 7537-7541. doi: 10.1128/AEM.01541-09

Sun, H., Xie, X., and Zu, G. R. (2010). Chelation of chitosan and its derivatives on soluble vitamin. Food Res. Dev. 31, 61-62.

Tsai, G. J., Zhang, S. L., and Shieh, P. L. (2004). Antimicrobial activity of a lowmolecular-weight chitosan obtained from cellulase digestion of chitosan. J. Food Protect. 67, 396-398. doi: 10.4315/0362-028X-67.2.396

Turner, J. L., Dritz, S. S., and Minton, J. E. (2001). Review: alternatives to conventional antimicrobials in swine diets. Prof. Anim. Sci. 17, 217-226. doi: 10.15232/S1080-7446(15)31633-8

Vahjen, W., Pietruszynska, D., Starke, I. C., and Zentek, J. (2015). High dietary zinc supplementation increases the occurrence of tetracycline and sulfonamide resistance genes in the intestine of weaned pigs. Gut Pathog 7:23. doi: 10.1186/s13099-015-0071-3

Varma, A. J., Deshpande, S. V., and Kennedy, J. F., (2004). Metal complexation by chitosan and its derivatives: a review. Carbohydr. Polym. 55, 77-93. doi: 10.1016/j.carbpol.2003.08.005

Vinsová, J., and Vavríková, E. (2011). Chitosan derivatives with antimicrobial, antitumour and antioxidant activities - a review. Curr. Pharmaceut. Design 17, 3596-3607. doi: 10.2174/138161211798194468

Wang, Q., Garrity, G. M., Tiedje, J. M., and Cole, J. R. (2007). Naive Bayesian classifer for rapid assignment of rRNA sequences into the new bacterial taxonomy. Appl. Environ. Microbiol. 78, 5261-5267. doi: 10.1128/AEM.00062-07
Wang, Y., Cao, P., Wang, L., Zhao, Z., Chen, Y., and Yang, Y. (2017). Bacterial community diversity associated with different levels of dietary nutrition in the rumen of sheep. Appl. Microbiol. Biotechnol. 101, 3717-3728. doi: 10.1007/s00253-017-8144-5

Wu, G. D., Chen, J., Hoffmann, C., Bittinger, K., Chen, Y. Y., Keilbaugh, S. A., et al. (2011). Linking long-term dietary patterns with gut microbial enterotypes. Science 334, 105-108. doi: 10.1126/science.1208344

Xiao, D. F., Wang, Y. F., Liu, G., He, J. H., Qiu, W., Hu, X., et al. (2014). Effects of chitosan on intestinal inflammation in weaned pigs challenged by enterotoxigenic Escherichia coli. PLoS ONE 9:e104192. doi: 10.1371/journal.pone.0104192

Xiong, X., Yang, H. S., Wang, X. C., Hu, Q., Liu, C. X., Wu, X., et al. (2015). Effect of low dosage of chito-oligosaccharide supplementation on intestinal morphology, immune response, antioxidant capacity, and barrier function in weaned piglets1. J. Anim. Sci. 93, 1089-1097. doi: 10.2527/jas.2014-7851

Yang, C. M., Ferket, P. R., Hong, Q. H., Zhou, J., Cao, G. T., Zhou, L., et al. (2012). Effect of chito-oligosaccharide on growth performance, intestinal barrier function, intestinal morphology and cecal micaoflora in weaned pigs. J. Anim. Sci. 90, 2671-2676. doi: 10.2527/jas.2011-4699

Yin, H., Du, Y. G., and Zhang, J. Z. (2009). Low molecular weight and oligomeric chitosans and their bioactivities. Curr. Topics Med. Chem. 9, 1546-1559. doi: 10.2174/156802609789909795

Yu, T., Zhu, C., Chen, S., Gao, L., Lv, H., Feng, R., et al. (2017). Dietary high zinc oxide modulates the microbiome of ileum and colon in weaned piglets. Front. Microbiol. 8:825. doi: 10.3389/fmicb.2017.00825

Zhou, T. X., Cho, J. H., and Kim, I. H. (2012). Effects of supplementation of chito-oligosaccharide on the growth performance, nutrient digestibility, blood characteristics and appearance of diarrhea in weanling pigs. Livest. Sci. 144, 263-268. doi: 10.1016/j.livsci.2011.12.009

Zhu, C., Lv, H., Chen, Z., Wang, L., Wu, X. J., Chen, Z. J., et al. (2017). Dietary zinc oxide modulates page range incldued antioxidant capacity, small intestine development, and jejunal gene expression in weaned piglets. Biol. Trace Elem. Res. 175, 331-338. doi: 10.1007/s12011-016-0767-3

Conflict of Interest Statement: The authors declare that the research was conducted in the absence of any commercial or financial relationships that could be construed as a potential conflict of interest.

Copyright (c) 2017 Yu, Wang, Chen, Hu, Wang, Wu, Ma, Chen and Zheng. This is an open-access article distributed under the terms of the Creative Commons Attribution License (CC BY). The use, distribution or reproduction in other forums is permitted, provided the original author(s) or licensor are credited and that the original publication in this journal is cited, in accordance with accepted academic practice. No use, distribution or reproduction is permitted which does not comply with these terms. 